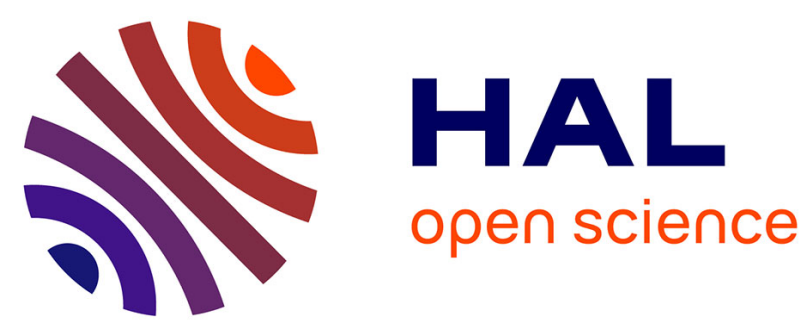

\title{
Diamond-coated ATR prism for infrared absorption spectroscopy of surface-modified diamond nanoparticles
}

Z. Remes, H. Kozak, B. Rezek, E. Ukraintsev, O. Babchenko, A. Kromka, Hugues Girard, Jean-Charles Arnault, P. Bergonzo

\section{- To cite this version:}

Z. Remes, H. Kozak, B. Rezek, E. Ukraintsev, O. Babchenko, et al.. Diamond-coated ATR prism for infrared absorption spectroscopy of surface-modified diamond nanoparticles. Applied Surface Science, 2013, 270, pp.411 - 417. 10.1016/j.apsusc.2013.01.039 . cea-01816405

HAL Id: cea-01816405

https://hal-cea.archives-ouvertes.fr/cea-01816405

Submitted on 30 Nov 2020

HAL is a multi-disciplinary open access archive for the deposit and dissemination of scientific research documents, whether they are published or not. The documents may come from teaching and research institutions in France or abroad, or from public or private research centers.
L'archive ouverte pluridisciplinaire HAL, est destinée au dépôt et à la diffusion de documents scientifiques de niveau recherche, publiés ou non, émanant des établissements d'enseignement et de recherche français ou étrangers, des laboratoires publics ou privés. 


\title{
Diamond-coated ATR prism for infrared absorption spectroscopy of surface-modified diamond nanoparticles
}

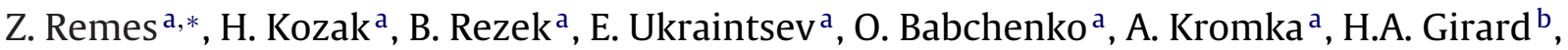 \\ J.-C. Arnault ${ }^{\mathrm{b}}$, P. Bergonzo ${ }^{\mathrm{b}}$ \\ a Institute of Physics of the ASCR, v.v.i., Cukrovarnicka 10, Praha 6, Czech Republic \\ b CEA, LIST, Diamond Sensors Laboratory, F-91191 Gif-sur-Yvette, France
}

\section{A R T I C L E I N F O}

\section{Article history:}

Received 6 August 2012

Received in revised form 7 December 2012

Accepted 7 January 2013

Available online 11 January 2013

\section{Keywords:}

ATR FTIR

CVD

Nanocrystalline diamond

Microwave

Hydrogenation

Nanopowder

\begin{abstract}
A B S T R A C T
Linear antenna microwave chemical vapor deposition process was used to homogeneously coat a $7 \mathrm{~cm}$ long silicon prism by $85 \mathrm{~nm}$ thin nanocrystalline diamond (NCD) layer. To show the advantages of the NCD-coated prism for attenuated total reflection Fourier transform infrared spectroscopy (ATR-FTIR) of nanoparticles, we apply diamond nanoparticles (DNPs) of $5 \mathrm{~nm}$ nominal size with various surface modifications by a drop-casting of their methanol dispersions. ATR-FTIR spectra of as-received, air-annealed, plasma-oxidized, and plasma-hydrogenated DNPs were measured in the $4000-1500 \mathrm{~cm}^{-1}$ spectral range. The spectra show high spectral resolution, high sensitivity to specific DNP surface moieties, and repeatability. The NCD coating provides mechanical protection against scratching and chemical stability of the surface. Moreover, unlike on bare Si surface, NCD hydrophilic properties enable optically homogeneous coverage by DNPs with some aggregation on submicron scale as evidenced by scanning electron microscopy and atomic force microscopy. Compared to transmission FTIR regime with $\mathrm{KBr}$ pellets, direct and uniform deposition of DNPs on NCD-ATR prism significantly simplifies and speeds up the analysis (from days to minutes). We discuss prospects for in situ monitoring of surface modifications and molecular grafting.
\end{abstract}

(c) 2013 Elsevier B.V. All rights reserved.

\section{Introduction}

Diamond nanoparticles (DNPs) are suitable for luminescence imaging, drug delivery or quantum engineering due to their optical transparency, mechanical and chemical stability, as well as low toxicity [1]. These applications require specific surface properties, as stability, affinity, targeting or therapeutic features, which are mainly conferred to the DNPs by an adapted surface chemistry [2]. The control of the surface termination is thus essential, with the homogeneous immobilization of different functional moieties such as carboxylic or alcoholic groups, $\mathrm{sp}^{2}$ species, or hydrogen terminations is the starting point to the functionalization and grafting of larger (bio-)molecules [3].

The mid-infrared optical absorption spectroscopy is commonly used to identify characteristic molecular vibrations [4]. Traditionally, the infrared optical absorption spectrum of powder sample is measured using Fourier Transform Infrared (FTIR) spectroscopy in a transmission mode after sample grinding to a fine powder and dispersing it in a $\mathrm{KBr}$ pellet. This method suffers from inevitable

\footnotetext{
* Corresponding author. Tel.: +420 220318516

E-mail address: remes@fzu.cz (Z. Remes).
}

reproducibility issues given the complexity of the sample preparation complicated by difficulties in getting sample homogenously dispersed throughout the $\mathrm{KBr}$ pellets. In addition, $\mathrm{KBr}$ is highly hydrophilic, and a drying procedure of the $\mathrm{KBr}$ pellet in situ the spectrometer is required in order to avoid water-related bands on the final IR spectrum.

The attenuated total reflection (ATR) spectroscopy is a well established FTIR technique for characterization of liquids, nonabrasive pastes, gels and deformable thin layers. This method is based on measuring the optical absorption of the evanescent wave that occurs in a totally internally reflected infrared beam when the sample is attached to the prism [5]. ATR offers faster sample preparation than transmission method and improves sample-to-sample reproducibility. Among other advantages of ATR belongs the ability to expose the sample to ambient atmosphere or chemicals to study in situ long-term stability and chemical reactivity. The ATR FTIR spectroscopy has been applied to monitor nanodiamond surface group transformations in the presence of aqueous solutions [6], however it has not yet been established as a widely used method to study of nano-powders such as diamond nanoparticles (DNPs). Indeed, the main difficulty of ATR is to achieve a good contact between the prism and the nano-powder while the standard ATR prism made of $\mathrm{ZnSe}$, Ge and Si easily scratches when the pressure 
is applied. This problem has been addressed recently by applying diamond prism [7]. However, diamond prisms are expensive and rather small, thus not allowing multiple reflections and often showing low sensitivity. The problematic is also an index of refraction of the diamond (2.4) since it is comparable to the index of refraction of the DNPs.

In this paper we employ nanocrystalline diamond (NCD) coating to significantly improve performance of conventional Si prism and to open new possibilities for (in situ) monitoring of diamond surface modifications. The growth of the thin, highly transparent nanocrystalline diamond (NCD) coatings deposited at a relatively low temperature with good adhesion on optical elements is still a challenge [8]. The technology of the NCD coating for optical applications on various substrates has been recently developed by our group using micro-wave chemical vapor deposition (MW CVD) [9]. To achieve large area, thin, uniform and high optical quality diamond coating, we employ previously developed technology for high diamond seeding densities [10] and large area plasma uniformity in linear antenna MW-CVD process [11]. We have shown that the non-diamond content in such coatings can be reduced by several orders of magnitude under optimized growth conditions followed by the post-deposition chemical etching and cleaning [12].

\section{Experimental}

The NCD coated silicon 6-reflection ATR prism has a length $72 \mathrm{~mm}$, width $10 \mathrm{~mm}$ and height $6 \mathrm{~mm}$. Prior the NCD coating growth on top of the prism, the Si surface was nucleated using ultrasound fine grained diamond powder (NanoAmando, New Metals and Chemicals Corp. Ltd., Kyobashi). The NCD coating was grown for several hours in the large area linear antenna MW CVD reactor (modified Roth \& Rau AK 400) from 0.5\% methane diluted in hydrogen at about $710^{\circ} \mathrm{C}$. Finally, the surface of the ATR prism was oxidized in RF plasma $(300 \mathrm{~W})$ for about $1 \mathrm{~min}$ to achieve the hydrophilic surface. The diamond character of NCD coating was confirmed by Raman spectroscopy (Renishaw In Via Reflex Raman UV spectrometer, excitation wavelength $325 \mathrm{~nm}$ ). The NCD thickness was evaluated from the reflectance spectra measured by the dual beam spectrometer equipped with Xe lamp as a light source and a UV-enhanced Si photodiode as a detector in the spectral region $250-1100 \mathrm{~nm}$.

Commercially available DNPs powder with the average nanoparticle size of $5 \mathrm{~nm}$ was purchased from NanoAmando, New Metals and Chemicals Corp. Ltd. (Kyobashi, Japan). The as-received DNPs were oxidized either $30 \mathrm{~min}$ in air at $450{ }^{\circ} \mathrm{C}$ or $10 \mathrm{~min}$ in RF plasma ( $300 \mathrm{~W}, 13.56 \mathrm{MHz})$. The hydrogenation has been done in hydrogen plasma generated in a quartz tube with a microwave power of $300 \mathrm{~W}$ and a pressure of $10 \mathrm{mbar}$ [13]. To prevent the contamination by air humidity and hydrocarbons of the organic origin, the DNPs were manipulated in a glove box purged with nitrogen atmosphere and stored dispersed in methanol.

Infrared absorption spectra were measured with a Thermo Nicolet 8700 FTIR spectrometer by two methods. First, in the standard transmittance mode using with diamond powder dispersed in $\mathrm{KBr}$ pellets, $150 \mathrm{mg} \mathrm{KBr}$ pellets were prepared with $3 \mathrm{mg}$ of DNPs. Up to $24 \mathrm{~h}$ before the measurement and during the acquisition, samples were kept under vacuum at $80^{\circ} \mathrm{C}$ to eliminate adsorbed water. Background signal was recorded via the pure $\mathrm{KBr}$ pellets placed next to the measured sample into the FTIR chamber. The transmittance method was using IR source, $\mathrm{KBr}$ beam-splitter and Peltier cooled pyrodetector (DTGS). Second, we applied the attenuated total reflectance (ATR) setup using Specac's Gateway ${ }^{\mathrm{TM}}$ 6-reflection horizontal ATR accessory with NCD coated ATR prism. The ATR method was applied using IR source, $\mathrm{KBr}$ beam-splitter and liquid
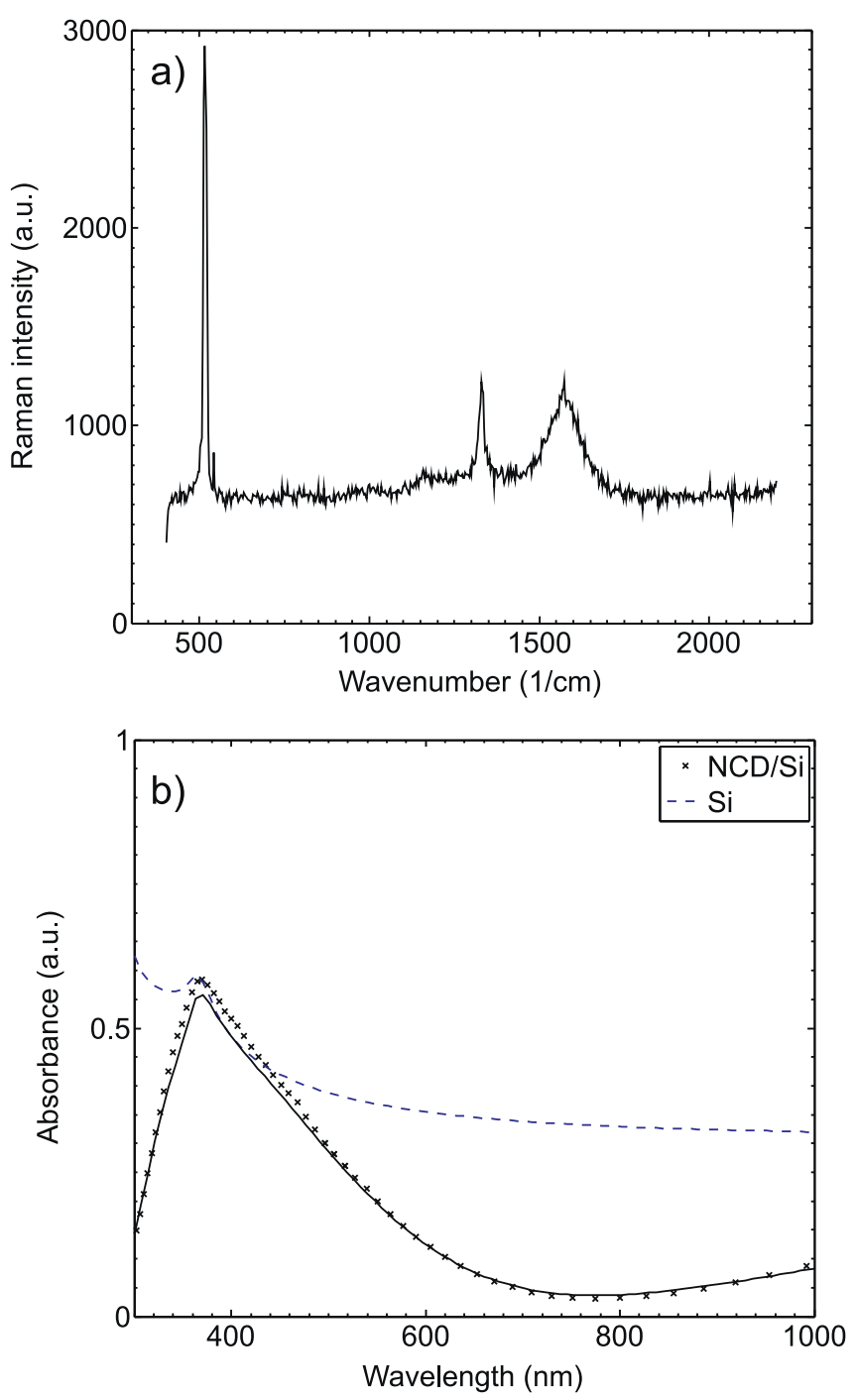

Fig. 1. (a) Raman spectra of NCD coated Si based ATR prism measured with the excitation wavelength $325 \mathrm{~nm}$. (b) The optical reflectance spectra of silicon covered with $85 \mathrm{~nm}$ thick diamond layer as well the theoretical fit (black solid curve). For comparison the calculated optical reflectance of Si was also added (blue dashed line). (For interpretation of the references to color in the artwork, the reader is referred to the web version of the article.)

nitrogen cooled photodiode (MCT) as a detector. Background spectrum was recorded using NCD-coated ATR prism without DNPs. The dispersion of $1 \mathrm{mg}$ DNPs in $2 \mathrm{ml}$ methanol was ultrasonicated for about $2 \mathrm{~h}$ before use, forming optically homogeneous dispersion The nanoparticles were applied on the ATR prism by drop-casting $70 \mu \mathrm{L}$ of methanol dispersion and letting dry. Methanol spreads well across the oxidized NCD surface. During the IR measurements the DNPs were in $\mathrm{N}_{2}$ purged chamber of the FTIR spectrometer. The ATR spectra were not measured below $1500 \mathrm{~cm}^{-1}$ where the $\mathrm{Si}$ prism is opaque. The advanced ATR correction was applied on the measured spectra. The ATR prism was mechanically cleaned by wet cotton buds followed by the control spectral measurement to prove that all absorbance peaks vanished from the spectrum.

The surface morphology and roughness of the diamond-coated prism before and after the deposition of DNPs was characterized by scanning electron microscopy (SEM, Raith e_LiNE) and atomic force microscopy (AFM, Veeco DI 3100 IV). Morphology measurements of DNPs were carried out in tapping mode using Multi75Al cantilevers. The measurements were repeated on ICON AFM in PeakForce QNM mode with similar results. The wear of Si tip on diamond substrate 
was observed. Yet its effect on the shape of measured particles, RMS roughness and coverage values was small. Note that due to NCD coating roughness AFM may not resolve all nanoparticles, in particular in small aggregates or individual.

The analysis of AFM data was performed using the Gwiddion software. The surface roughness was calculated based on entire $5 \times 5 \mu \mathrm{m}^{2}$ image. The analysis of smaller area and the measurements in different area with smaller or larger amount of aggregates of DNPs yield error bar of RMS roughness $\pm 5 \mathrm{~nm}$. Surface coverage by diamond particles was estimated from AFM image using threshold equal to the average height value of the surface plus $3 \times$ RMS roughness of bare ATR prism. The average height value was used as basic offset. Additional offset of $3 \times$ RMS roughness of bare ATR prism was used to minimize influence of the ATR prism roughness. The objects with height above this threshold are attributed to nanoparticles and marked with red color. Surface coverage is a ratio of the area marked with red color and the whole image area. The estimated residual coverage of NCD coated ATR prism by this method is used as an error bar. The calculated surface coverage is a bottom estimate because such threshold possibly neglects small DNPs with size lower than roughness of ATR prism.

\section{Results}

\subsection{Characterization of the diamond ATR coated prism}

The Raman spectrum of NCD-coated Si ATR prism shown in Fig. 1 a reveals a strong peak at $520 \mathrm{~cm}^{-1}$ related to the $S i$ prism and a peak at about $1330 \mathrm{~cm}^{-1}$ related to $\mathrm{sp}^{3}$ carbon of the diamond crystallites. This proofs the diamond character of the NCD coating on Si prism. A broad G band centered at $1570 \mathrm{~cm}^{-1}$ is related to the non-diamond content and especially to the residual $\mathrm{sp}^{2}$ contamination at grain boundaries of our NCD layer. Note that similar Raman spectra were recorded all over the sample, demonstrating the homogeneity of the NCD coating.

In order to estimate the index of refraction and the thickness of the diamond coating, the optical reflectance spectra in the spectral region 300-1000 $\mathrm{nm}$ were fitted with a single layer on Si substrate using the FilmWizard commercial software, see Fig. 1b. The index of refraction was found to follow the Cauchy formula $2.38+0.02 / \lambda^{2}$, where $\lambda$ is the wavelength in $\mu \mathrm{m}$ and the thickness about $85 \mathrm{~nm}$ of NCD coating on Si prism have been estimated.

Fig. 2(a) and (b) shows SEM images of the clean NCD-coated ATR prism under various magnification. The SEM images are typical across the whole prism area. They evidence uniformity and continuity of the coating. The size of nanocrystals in the NCD layer is in the order of tens of $\mathrm{nm}$ as estimated from the SEM images in Fig. 2(a) and (b). SEM images in Fig. 2(c) and (f) shows the NCD-coated ATR prism surface with the DNPs drop-casted from methanol dispersion. There are some additional darker features compared to the clean prism. However, it is difficult to clearly identify the DNPs on diamond coating in the SEM images.

In order to examine DNPs aggregation and coverage, the AFM images recorded and analyzed in detail. Fig. 3a illustrates the method used to calculate the bottom estimate of surface coverage of ATR prism by diamond nanoparticles. The detailed procedure is described in the experimental part. Fig. 3b shows that indeed on bare prism surface only few objects are (falsely) identified as DNPs using the method. Estimated surface coverage of $1 \%$ is due to slightly higher roughness in several places and it can be used as an error bar of the method. Fig. 3c shows that as-received DNPs homogeneously cover ATR prism and form small aggregates with $200-500 \mathrm{~nm}$ width. Coverage is higher than $16 \%$, RMS roughness is $27 \pm 5 \mathrm{~nm}$ compared to $7 \pm 1 \mathrm{~nm}$ of the bare NCD surface. Fig. 3d shows that oxygen termination of DNPs causes formation of larger aggregates with $1 \mu \mathrm{m}$ in width and $100-200 \mathrm{~nm}$ in height. The coverage increases to $23 \%$, but the number of aggregates decreases compare to the as-received DNPs. The RMS roughness also increased to $32 \pm 5 \mathrm{~nm}$. Fig. 3e demonstrates that annealing in air of DNPs also leads to similar formation of larger aggregates with $1 \mu \mathrm{m}$ width and $100-200 \mathrm{~nm}$ height. The coverage is $18 \%$, RMS roughness is $28 \pm 5 \mathrm{~nm}$. On the other hand, hydrogen termination causes reduction of RMS roughness $(20 \pm 5 \mathrm{~nm})$ and coverage ( $8 \%$ ) compared to as-received DNPs. Moreover, there are only few large aggregates of DNPs (images without large aggregates were also obtained, data not shown). The skew of surface height on all images is higher than zero. This means that the size distribution on DNPs is not symmetrical, i.e. some aggregation of DNPs occurs in all cases. Table 1 summarizes the values of surface coverage, RMS roughness and skew.

For comparison, Fig. 4 shows the DNPs surface coverage of bare silicon surface plasma oxidized one day prior to the DNPs drop-casting from methanol dispersion. As-received and hydrogenated DNPs spread relatively homogeneously compared to the wavelength of the mid-IR light $(2-20 \mu \mathrm{m})$, whereas others such as plasma-oxidized DNPs show low affinity to the silicon surface resulting in the more pronounced aggregation and inhomogeneities compared to the NCD-coated prism.

\subsection{FTIR characterization of the DNPS on NCD coated ATR prism}

Fig. 5 shows the normalized IR absorbance spectra of the asreceived, air-annealed, plasma-hydrogenated and plasma-oxidized DNPs measured in the ATR mode at room temperature in nitrogen atmosphere. The as-received DNPs ATR spectrum is dominated by a large carboxyl-related band centered around $1700 \mathrm{~cm}^{-1}$, the $\mathrm{C}-\mathrm{H}$ stretching band centered at $2800-3000 \mathrm{~cm}^{-1}$ and $\mathrm{O}-\mathrm{H}$ stretching band at $3000-3600 \mathrm{~cm}^{-1}$ (related with carboxyl groups of DNPs and/or water). As expected after the hydrogenation treatment, most of the oxygenated terminations are removed and the spectrum is dominated by clearly distinguishable $\mathrm{C}-\mathrm{H}$ peaks at 2856 , 2872,2925 and $2958 \mathrm{~cm}^{-1}$. On the other hand, after the oxidation by oxygen plasma or by annealing in air, the $\mathrm{C}-\mathrm{H}$ peaks disappear, while carboxyl related bands at $3200-3400 \mathrm{~cm}^{-1}$ (-OH stretching) and $1800 \mathrm{~cm}^{-1}$ the $(>C=0)$ are observed.

As a comparison, plasma oxidized NDs were studied in transmittance mode, dispersed in $\mathrm{KBr}$ pellet. Fig. 6 shows the evolution of the FTIR spectra according to the annealing $\left(80^{\circ} \mathrm{C}\right)$ duration, performed to remove adsorbed water. As shown by the spectrum recorded before annealing, water adsorbed in the $\mathrm{KBr}$ pellet strongly interfere with the signature of the plasma treated NDs, with two major contributions at 3500 and $1630 \mathrm{~cm}^{-1}$. Thanks to the in situ annealing, these two bands progressively vanish with time. At least $24 \mathrm{~h}$ of drying is required to obtain a spectrum comparable to the one obtained by ATR mode, on which similar peaks are observed, with the presence of carboxylic-related bands and some $\mathrm{C}-\mathrm{H}$ stretching.

\section{Discussions}

The oxygen plasma treatment of the diamond coated ATR surface significantly reduces contact angle and gives rise to a strong affinity to polar solvents [14]. As a result, methanol spreads and evaporates uniformly on the oxidized NCD surface, leaving some sub-micrometer aggregates of the functionalized DNPs adsorbed on the whole ATR prism surface. Such coverage is optically homogeneous for FTIR wavelength. We suppose that the self-assembly and adhesion of the DNPs applied on ATR prism by drop-casting is an electrostatic process (physisorption) which can be controlled by the surface groups of the ATR prism coatings and the solution. We found the interaction to be quite strong, so the adsorbed DNPs 

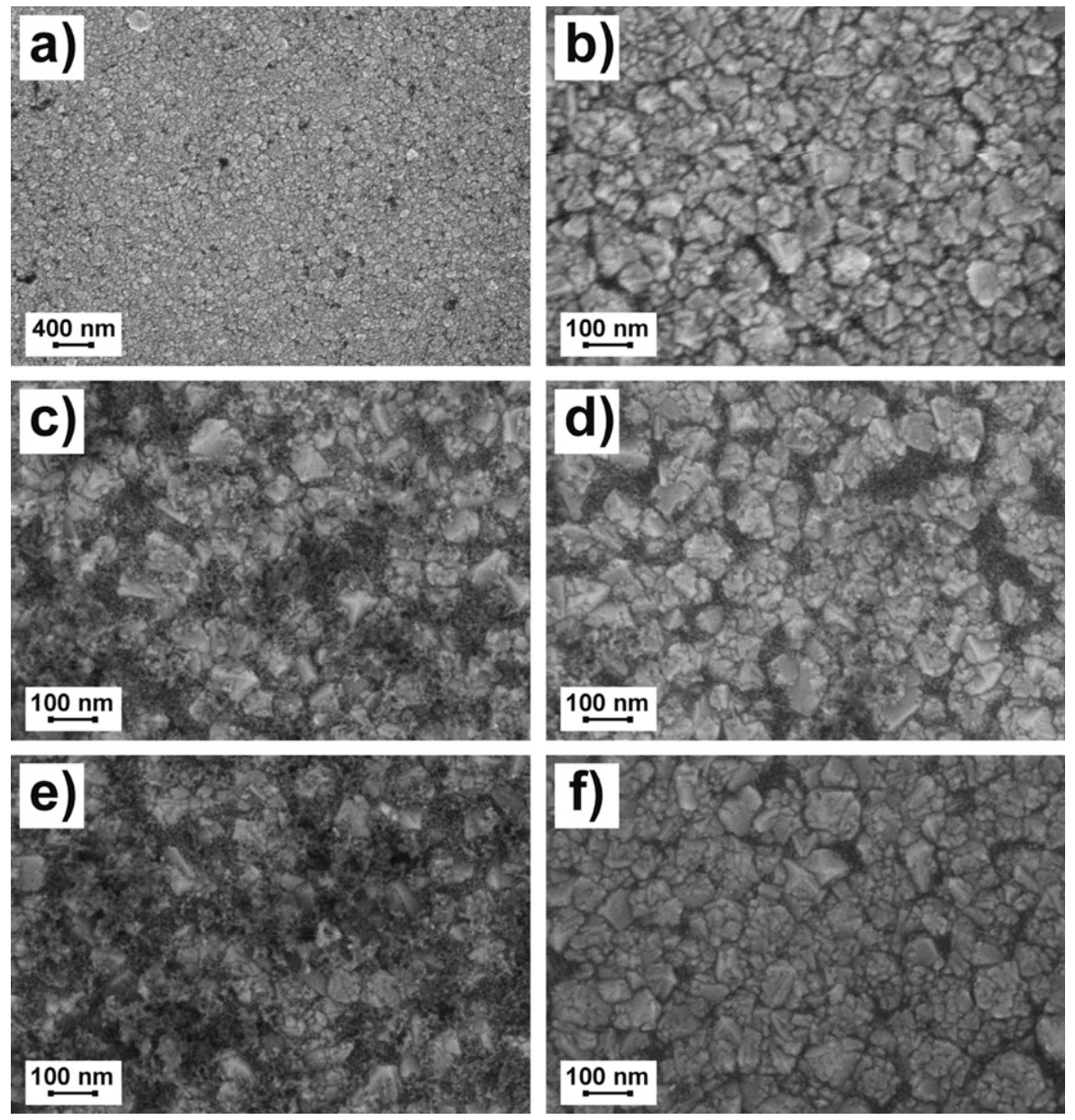

Fig. 2. SEM images of NCD coated ATR prism: (a) and (b) without and with DNPs, (c) as-received, (d) plasma oxidized, (e) annealed in air, and (f) plasma hydrogenated DNPs.

cannot be easily removed by air blow or water rinsing. Yet, they can be removed mechanically by wiping with wet cotton buds and the FTIR prism can be repeatedly re-used with the same spectral quality.

The AFM analysis reveals that the height histogram is asymmetrical with positive skew. The main cause can be the presence of sub-micron DNP aggregates. Due to positive skew the average height value is higher than the height corresponding to the maximum of the histogram, as shown in Fig. 3a. In the case of identical nanoparticles skew should be around zero. For example, high skew value of hydrogenated DNPs on ATR prism (5.2) is due to presence of only two large aggregates of DNPs. Without them, skew will be $\sim 1.5$, RMS roughness will be around $11 \pm 2 \mathrm{~nm}$, and surface coverage around $4 \%$.

From AFM it is obvious that oxygen-terminated DNPs tend to form larger aggregates compared to the as-received and in particular H-terminated DNPs. This can be due to etheric type linkages between DNPs [15]. Hydrogen termination reduces aggregation and size of aggregates even compared to the as-received DNPs. This is probably due to less reactive surface and H-plasma removal of graphitic shells typical for the as-received DNPs [16].

The diamond coated ATR prism demonstrates here its high sensitivity for hydrogenated terminations, as $\mathrm{C}-\mathrm{H}$ vibrations seem to be exalted. Indeed, these $\mathrm{C}-\mathrm{H}$ stretching bands are expected on hydrogenated NDs, and their presence is not excluded on as-received DNPs carrying amorphous carbon. Nevertheless, ATR spectra also reveal the strong decrease of the $\mathrm{C}-\mathrm{H}$ stretching bands after air annealing or plasma treatment, expected after such oxidation processes. We can see that ATR-FTIR using diamond-coated prism provides a similar spectral resolution of DNP surface moieties as the FTIR in transmission mode. Slight difference is given by the use of different detector types rather than by specifics of each regime. The signal is somewhat noisier in the ATR regime because of lower amount of DNPs on the prism surface compared to the $\mathrm{KBr}$ pellet. Yet the diamond-coated ATR prism provides high sensitivity to H-termination of DNPs. The hydrogen-related peaks are most pronounced on the plasma-hydrogenated and as-received DNPs. MW plasma provides well-defined H-termination on DNPs

Table 1

The root mean square (RMS) roughness of the surface and the bottom estimate of the DNPs surface coverage on the ATR prism (as shown in Fig. 3).

\begin{tabular}{|c|c|c|c|}
\hline Sample & RMS roughness (nm) & Coverage (\%) & Skew \\
\hline NCD coated ATR prism & 7 & 1 & 1.0 \\
\hline NCD coated ATR prism with as-received DNPs & 27 & 16 & 2.1 \\
\hline NCD coated ATR prism with oxidized DNPs & 32 & 23 & 1.0 \\
\hline NCD coated ATR prism with annealed in air DNPs & 28 & 18 & 1.8 \\
\hline NCD coated ATR prism with hydrogenated DNPs & 20 & 8 & 5.2 \\
\hline
\end{tabular}



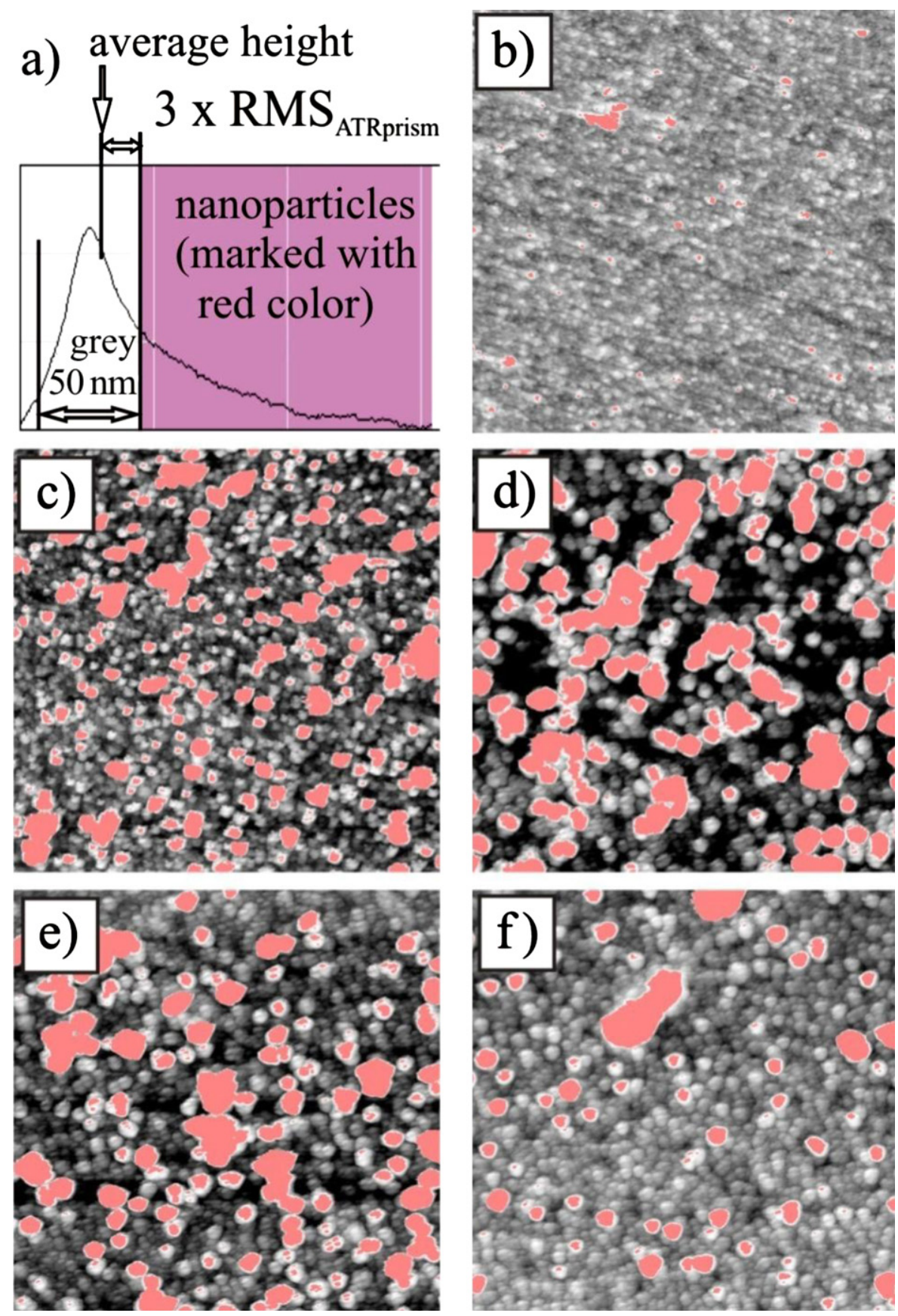

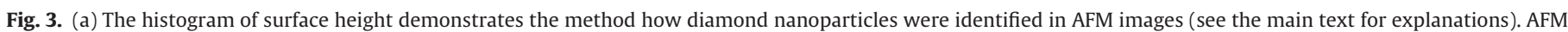

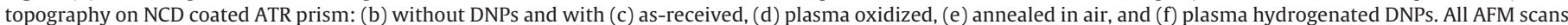

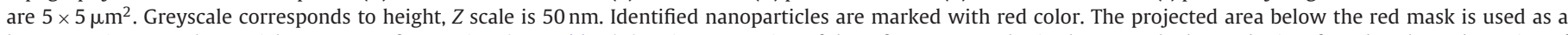

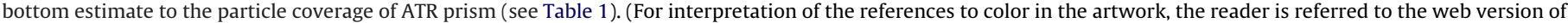
the article.)

surfaces, stable up to $600{ }^{\circ} \mathrm{C}$ in vacuum, up to $350^{\circ} \mathrm{C}$ in air and up to $100^{\circ} \mathrm{C}$ in strong acids [17]. As expected, a strong decrease of the oxygen-carbon related bands occurs after the hydrogenation, linked to the desorption of the oxygenated terminations, and the saturation of the dangling bonds with hydrogen [16]. Thus the $\mathrm{C}-\mathrm{H}$ stretching bands are due to $\mathrm{H}$-termination of diamond surface in this case. These $\mathrm{C}-\mathrm{H}$ stretching bands can also be related to amorphous carbon, too. This is probably the case on as-received DNPs. The wet chemical treatment in strong acids at elevated temperatures, which is used for as-received DNPs cleaning after a fabrication process, leads typically to their carboxylation yet some amorphous carbon shells remain [18].
Thermal annealing in air at $400-500^{\circ} \mathrm{C}$ leads to oxidation with carboxylic groups and selective etching of non-diamond carbon [19]. Similarly, oxygen plasma efficiently removes hydrogen from diamond surface, etches the non-diamond phase and give rise to mostly $\mathrm{C}=\mathrm{O}$ and $\mathrm{C}-\mathrm{O}-\mathrm{C}$ moieties rather than carboxylic groups [20]. Such effects are clearly detected in the ATR-FTIR spectra in Fig. 5.

Thus the ATR-FTIR is able to detect and monitor various surfaces moieties of DNPs with a good sensitivity. Unlike the standard transmission mode that requires up to $24 \mathrm{~h} \mathrm{KBr}$ annealing to get a water-free spectrum, ATR spectrum is recorded in several minutes. NCD coated ATR prism offers several other advantages: 

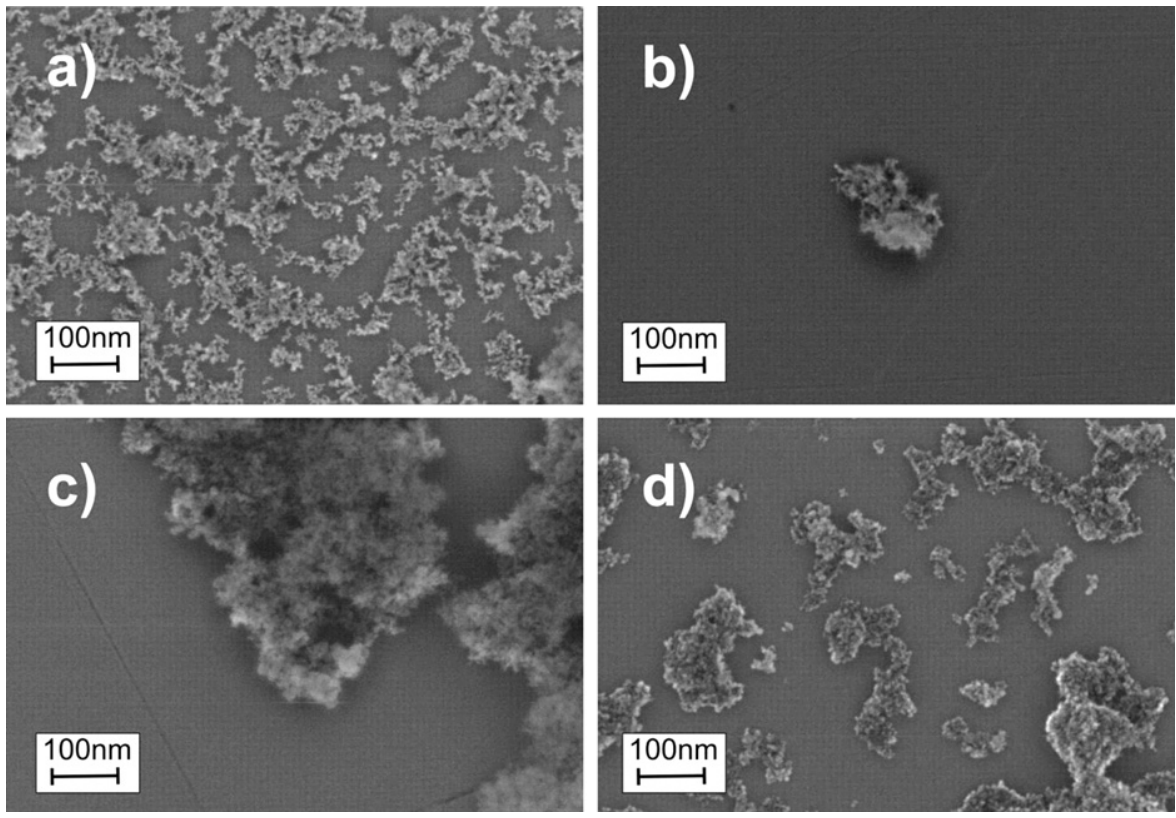

Fig. 4. SEM images of silicon surface coated with the (a) as-received, (b) plasma oxidized, (c) annealed in air, and (d) plasma hydrogenated DNPs.

mechanical robustness, the stable hydrophobicity/hydrophilicity that can be tuned for studies of proteins and other biomolecules [21], inertness of the material to heat or gas treatments [22], as well as the biocompatibility of diamond [23]. The diamond ATR coating may also provide new insights on the diamond chemistry due possible in situ monitoring of any kind of reactions performed on surface-adsorbed nanoparticles or directly on diamond thin films. For instance, starting from hydrogenated layers, one may be able to monitor the grafting of alkenes under UV, diazonium chemistry, etc. [24,25] as well as the intermediate species usually hardly accessible with common techniques. As diamond is promised to be an exceptional biointerface, ATR can also be an valuable tool to validate and investigate biochemical grafting, or even more

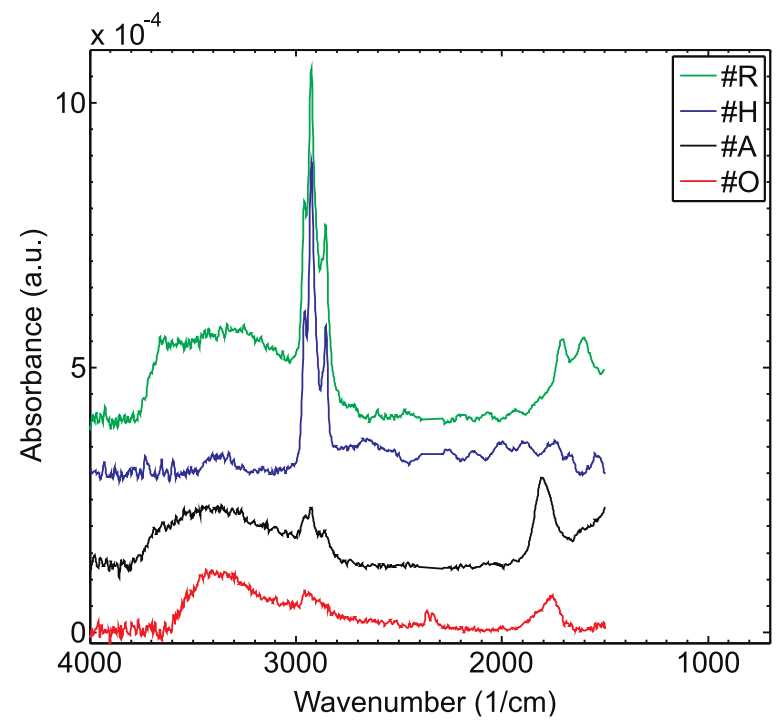

Fig. 5. IR absorbance spectra (in the absorbance units) of the as received (\#R), plasma hydrogenated (\#H), annealed in air (\#A) and plasma oxidized (\#O) diamond nanoparticles measured at room temperature in nitrogen atmosphere using the ATR mode. The spectra were normalized and stacked for clarity. In the region below $1500 \mathrm{~cm}^{-1}$ silicon prism does not transmit IR radiation and so there is no spectral information available in this region. (For interpretation of the references to color in the artwork, the reader is referred to the web version of the article.)

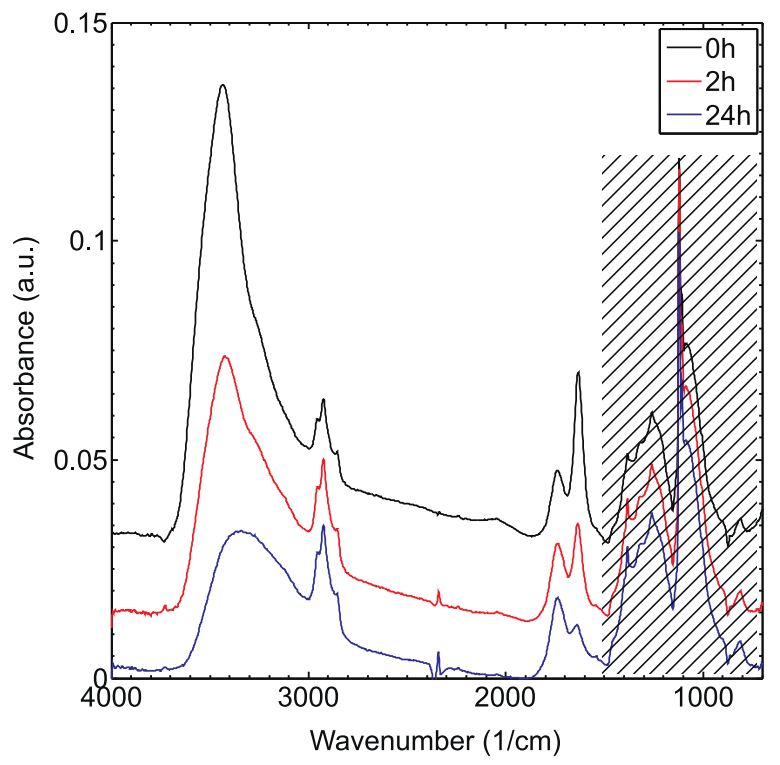

Fig. 6. IR absorbance spectra (in the absorbance units) of the plasma oxidized diamond nanoparticles measured at $80^{\circ} \mathrm{C}$ in vacuum in transmittance mode with diamond powder dispersed in $\mathrm{KBr}$ pellets. The spectra were annealed in vacuum at $80^{\circ} \mathrm{C}$ for 0,2 and $24 \mathrm{~h}$. The spectra were normalized and stacked for clarity. (For interpretation of the references to color in the artwork, the reader is referred to the web version of the article.)

interactions with biological species. The limiting point of the present technique is the lack of information in the region below $1500 \mathrm{~cm}^{-1}$, because of absorption in silicon prism. This can be avoided by using a diamond-coated Ge prism.

\section{Conclusions}

We demonstrated the usability and the accuracy of the nanocrystalline diamond (NCD) coated ATR prism as a novel tool for FTIR spectroscopy of surface modified nano-particles, in particular DNPs with various surface moieties and we compared these results with spectra obtained by standard transmittance method based on dispersing the DNPs in KBr pellets [13] and others FTIR 
techniques to follow functional group chemistry of DNPs [26]. NCD coated ATR prism successfully detected various hydrogen and oxygen moieties on air-annealed and plasma-treated DNPs. Essential part was the use and optimization of the linear antenna CVD for NCD thin film coating on the Si ATR prism. The NCD coating provides several fundamental advantages for ATR-FTIR: (1) mechanical protection of the ATR prism surface against scratching which enables reliable repeatable use and comparison of the spectra, (2) chemically stable surface for revealing possible changes of nanoparticles in time, (3) stable hydrophilic surface which leads to optically homogeneous coverage and good adhesion of nanoparticles dropcasted from polar solvents (such as methanol), (4) ATR spectrum is recorded in several minutes unlike the standard transmission mode that requires up to $24 \mathrm{~h} \mathrm{KBr}$ annealing to get a water-free spectrum. In broader perspective, the diamond ATR coating may also provide new insights on the diamond chemistry due possible in situ monitoring of any kind of reactions performed on diamond thin films.

\section{Acknowledgements}

We acknowledge the financial support by the following projects: M100100902, P205/12/P331, P108/12/0910, Fellowship J. E. Purkyne, and MSMT KONTAKT II projects LH12186 and LH12236. The work occurred in frame of LNSM infrastructure.

\section{References}

[1] V.N. Mochalin, O. Shenderova, D. Ho, Y. Gogotsi, Nature Nanotechnology 7 (2011) 11-23.

[2] E. Ōsawa, Diamond and Related Materials 16 (2007) 2018-2022.

[3] A. Krueger, D. Lang, Advanced Functional Materials 22 (2012) 890-906.

[4] G. Socrates, Infrared and Raman Characteristic Group Frequencies: Tables and Charts, J. Wiley, Chichester, 2001.

[5] N.J. Harrick, Internal Reflection Spectroscopy, John Wiley \& Sons Inc., New York, 1967.
[6] J. Scholz, A.J. McQuillan, K.B. Holt, Chemical Communications 47 (2011) 12140.

[7] H.A. Girard, S. Perruchas, C. Gesset, M. Chaigneau, L. Vieille, J.-C. Arnault, P. Bergonzo, J.-P. Boilot, T. Gacoin, ACS Applied Materials \& Interfaces 1 (2009) 2738-2746.

[8] Z. Remes, H. Kozak, O. Babchenko, S. Potocky, E. Ukraintsev, B. Rezek, A. Kromka, Diamond and Related Materials 20 (2011) 882-885.

[9] A. Kromka, O. Babchenko, T. Izak, K. Hruska, B. Rezek, Vacuum 86 (2012) 776-779.

[10] A. Kromka, O. Babchenko, H. Kozak, K. Hruska, B. Rezek, M. Ledinsky, J. Potmesil, M. Michalka, M. Vanecek, Diamond and Related Materials 18 (2009) 734-739.

[11] K. Tsugawa, S. Kawaki, M. Ishihara, J. Kim, Y. Koga, H. Sakakita, H. Koguchi, M. Hasegawa, Diamond and Related Materials 20 (2011) 833-838.

[12] Z. Remes, T. Izak, A. Kromka, M. Vanecek, Diamond and Related Materials 19 (2010) 453-456.

[13] H.A. Girard, J.C. Arnault, S. Perruchas, S. Saada, T. Gacoin, J.-P. Boilot, P. Bergonzo, Diamond and Related Materials 19 (2010) 1117-1123.

[14] B. Rezek, M. Kratka, E. Ukraintsev, O. Babchenko, A. Kromka, A. Broz, M. Kalbacova, in: P.A. Serra (Ed.), New Perspectives in Biosensors Technology and Applications, InTech, Vienna, Austria, 2011, p. 448.

[15] I. Petrov, O. Shenderova, V. Grishko, V. Grichko, T. Tyler, G. Cunningham, G. McGuire, Diamond and Related Materials 16 (2007) 2098-2103.

[16] J.-C. Arnault, T. Petit, H. Girard, A. Chavanne, C. Gesset, M. Sennour, M. Chaigneau, Physical Chemistry Chemical Physics 13 (2011) 11481-11487.

[17] H.A. Girard, T. Petit, S. Perruchas, T. Gacoin, C. Gesset, J.C. Arnault, P. Bergonzo, Physical Chemistry Chemical Physics 13 (2011) 11517.

[18] K. Takahashi, M. Tanga, O. Takai, H. Okamura, Diamond and Related Materials 12 (2003) 572-576.

[19] S. Osswald, G. Yushin, V. Mochalin, S.O. Kucheyev, Y. Gogotsi, Journal of the American Chemical Society 128 (2006) 11635-11642.

[20] P.E. Pehrsson, T.W. Mercer, Surface Science 460 (2000) 49-66.

[21] B. Rezek, L. Michalíková, E. Ukraintsev, A. Kromka, M. Kalbacova, Sensors 9 (2009) 3549-3562.

[22] M. Davydova, A. Kromka, P. Exnar, M. Stuchlik, K. Hruska, M. Vanecek, M. Kalbac, Physica Status Solidi (a) 206 (2009) 2070-2073.

[23] L. Bacakova, L. Grausova, J. Vacik, A. Kromka, H. Biederman, A. Choukourov, V. Stary, in: B. Reddy (Ed.), Advances in Diverse Industrial Applications of Nanocomposites, InTech, Vienna, Austria, 2011, pp. 399-436.

[24] C.E. Nebel, D. Shin, B. Rezek, N. Tokuda, H. Uetsuka, H. Watanabe, Journal of the Royal Society Interface 4 (2007) 439-461.

[25] B. Rezek, D. Shin, H. Uetsuka, C.E. Nebel, Physica Status Solidi (a) 204 (2007) 2888-2897.

[26] J. Zhang, D.S. Su, R. Blume, R. Schlögl, R. Wang, X. Yang, A. Gajović, Angewandte Chemie International Edition 49 (2010) 8640-8644. 